\title{
Platelet activation and aggregation profile in prolonged external ventricular support
}

\author{
Rémi Houël, $M D^{\mathrm{a}}$ \\ Elisabeth Mazoyer, PharmD ${ }^{b}$ \\ Bernadette Boval, PharmD ${ }^{\mathrm{b}}$ \\ Mathias Kirsch, MDa \\ Emmanuelle Vermès, $M D^{\mathrm{a}}$ \\ Ludovic Drouet, PharmD ${ }^{\mathrm{b}}$ \\ Daniel Y. Loisance, $\mathrm{MD}^{\mathrm{a}}$
}

\footnotetext{
From the Service de chirurgie cardiaque, Hôpital Henri Mondor, ${ }^{a}$ Créteil, France, Unité, Association Claude Bernard and Service d'hématologie biologique, Hôpital Lariboisière, ${ }^{\mathrm{b}}$ Paris, France.

Received for publication July 28, 2003; revisions received Oct 20, 2003; accepted for publication Nov 17, 2003.

Address for reprints: Rémi Houël, Institut Jacques Cartier, Adult Cardiac Surgery, 6 Avenue du Noyer Lambert, Massy 91300, France (E-mail: remihouel@hotmail.com).

J Thorac Cardiovasc Surg 2004;128:197-202

0022-5223/\$30.00

Copyright (C) 2003 by The American Association for Thoracic Surgery

doi:10.1016/j.jtcvs.2003.11.059
}

Background: Platelet function plays a major role in the understanding of thromboembolic events in prolonged mechanical support. We studied the platelet activation, platelet aggregation profile, and efficacy of aspirin in patients in whom an external ventricular assist device had been implanted.

Patients and Methods: Fifteen patients were studied prospectively up to 6 weeks after implantation of the same type of ventricular assist device. Platelet function was studied weekly before daily aspirin administration. Aspirin efficacy was tested ex vivo by measuring platelet aggregation triggered by arachidonic acid. Flow cytometry was used to quantify the spontaneous and induced (adenosine diphosphate stimulation) expression of glycoproteins $\alpha \mathrm{IIb} \beta 3, \mathrm{Ib} \alpha$, and $\mathrm{CD} 62 \mathrm{P}$ on platelet membranes. The plasma levels of von Willebrand factor (von Willebrand factor activity and von Willebrand factor antigen) and fibrinogen were also determined.

Results: Six of the 15 patients (26\%) maintained an arachidonic acid-induced platelet aggregation despite daily aspirin treatment $(250 \mathrm{mg})$. CD62P values remained increased during a 5-week postoperative period. Spontaneous levels of glycoproteins $\alpha \operatorname{IIb} \beta 3$ and $\operatorname{Ib} \alpha$ on platelet membranes remained within a normal range with a preserved reactivity. The plasma levels of fibrinogen and von Willebrand factor remained increased during the entire study period.

Conclusion: In patients with an implanted external ventricular assist device, the platelet activation profile displays a persistent activation with a preserved reactivity associated with a persistent high inflammatory state and endothelial activation.

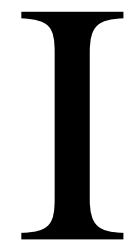

n patients with an external ventricular assist device (VAD), anticoagulation protocols, such as heparin or antivitamin $\mathrm{K}$ anticoagulation, are usually associated with at least 1 antiplatelet agent. Aspirin is the most frequently used antiplatelet agent despite its individual variable effect on ex vivo and in vivo platelet aggregation. ${ }^{1,2}$ Some resistance to aspirin has been observed recently with the use of low doses of aspirin in patients with atherosclerosis or after cardiopulmonary bypass (CPB) in coronary artery bypass grafting. ${ }^{3,4}$ The explanation for this so-called resistance to aspirin is multiple: (1) an increased platelet turnover after $\mathrm{CPB}$, (2) cyclooxygenase-2 induction associated with an inflammatory state, and (3) other sources of thromboxane $\mathrm{A}_{2}$ production insensitive to aspirin. ${ }^{3-5}$ However, our knowledge on platelet function in patients with an implanted VAD remains limited.

Platelet activation is best tested by a flow cytometry technique that measures the expression of integrins on the platelet membrane surface. ${ }^{6,7}$ Integrins $\alpha \operatorname{IIb} \beta 3$ and $\mathrm{Ib} \alpha$ seem to play a key role in platelet activation and thrombus formation. Integrin 
$\alpha \operatorname{IIb} \beta 3$ is the last final common pathway of platelet aggregation through its binding to fibrinogen or von Willebrand factor. ${ }^{8}$ Integrin $\mathrm{Ib} \alpha$ mediates platelet activation and adhesion through its binding to von Willebrand factor on the subendothelium, especially in high shear stress flowing conditions. $^{9}$

The clinical development of VAD has brought some changes in hemodynamic biomaterials or conduits to reduce the incidence of device-related thromboembolic events. These improvements could also be explained by a more favorable host response to biomaterial implantation. ${ }^{10}$ These results may also illustrate different patterns of host response to different devices in which surface texture and hemodynamic conditions are different. ${ }^{11}$ The experience gained during the last decade in mechanical support allows studies on homogenous populations with a single type of support and prolonged support time.

This report studies (1) the platelet activation and aggregation profile of patients with a single type of external VAD and (2) the biologic efficacy of aspirin in these patients during prolonged support.

\section{Patients and Methods}

\section{Patient Selection}

Fifteen patients were studied prospectively during a 6-week period after implantation of the same type of external VAD (Thoratec Corporation, Pleasanton, Calif).

The indications for emergency device implantation were the following: persistent primary cardiogenic shock complicating acute myocardial infarction ( 7 patients), acute myocarditis ( 2 patients), and end-stage dilated cardiomyopathy (6 patients). Seven of the 15 patients were supported by an intra-aortic balloon pump before device implantation.

\section{Surgical Technique and Anticoagulation Protocol}

Implantation was performed in a standard technique with $\mathrm{CPB}$ and moderate hypothermia. The left inflow cannula was implanted through the left ventricular apex in all instances. The right inflow cannula was implanted through the right atrium in all cases except 2 (into the right ventricle). The device was run in a fill-to-empty mode in all instances and as soon as possible (within hours postoperatively) on the left side. On the right side, the device was run in a fill-to-empty mode but could be on a fixed mode because of insufficient filling (postoperatively) or right ventricular recovery.

After surgery, anticoagulation was started within 8 to 12 hours using intravenous heparin to achieve an anti-Xa activity between 0.3 and $0.4 \mathrm{IU} / \mathrm{L}$. Aspirin was started 24 hours after surgery at a daily dose of $250 \mathrm{mg}$. Aspirin doses were then increased gradually from $250 \mathrm{mg}$ to $350 \mathrm{mg}$ when ex vivo platelet function tests showed a persistent aggregation in the presence of arachidonic acid (AA) and finally up to $500 \mathrm{mg}$ per day if ex vivo aggregation persisted after the previous increased dosage. In case of active bleeding with no obvious surgical source or neurologic bleeding complication, aspirin administration was stopped. Antivitamin K therapy was started after removal of all chest drains and extubation to maintain the international normalized ratio between 3 and 4 . Fresh blood was collected for platelet function studies and cytometric analysis was performed weekly from the week of implantation and before daily aspirin administration. Blood was collected from a peripheral vein using a Vacutainer system (Becton Dickinson, Franklin Lakes, NJ) (as for control patients) and immediately stored in ice for transport to the laboratory. The dosage of von Willebrand factor, plasmatic fibrinogen levels, and platelet count were determined in the same conditions.

\section{Resistance to Aspirin}

A resistance to aspirin treatment was defined by any persistent ex vivo platelet aggregation induced by AA despite a daily administration of aspirin.

The platelet count was determined on an ethylenediaminetetraacetic acid blood sample using an STKS counter (Beckman Coulter France, Paris, France).

\section{Platelet Aggregation}

The citrated blood sample was centrifuged at $200 \mathrm{~g}$ for 10 minutes at room temperature. Platelet-rich plasma (PRP) was separated, and platelet aggregation was monitored by the turbidimetric method on a Chronolog Aggregometer (Beckman Coulter). PRP was stirred at $37^{\circ} \mathrm{C}$ for 1 minute under constant agitation $(1000$ rpm) and aggregation was induced by the addition of AA (0.2 $\mathrm{mg} / \mathrm{mL}$ final concentration, Sigma, Lyon, France). Velocity and maximal intensity were recorded, and the results are expressed as the percentage of aggregation.

The plasma fibrinogen level was determined using a Clauss technique (Fibromat, BioMerieux, Paris, France).

The von Willebrand factor was determined using an enzymelinked immunosorbent assay technique for the antigenicity (Asserachrom vWF; Diagnostica Stago, Asnières, France) and ristocetin-induced platelet aggregation for the activity (von Willebrand reagent; Dade Behring, Paris, France).

\section{Flow Cytometric Analysis}

To study platelet activation, we measured the spontaneous expression of CD62P (P-selectin or granule membrane protein 140) on the platelet membrane surface. Platelet activability was assessed by measuring the membrane expression of glycoproteins (GPs) $\alpha \mathrm{IIb} \beta 3$ and $\mathrm{Ib} \alpha$ in the absence and presence of adenosine diphosphate (ADP) (Beckman Coulter). Ex vivo, diluted PRP (40 $\mu \mathrm{L})$ was incubated in the absence or presence of ADP $(25 \mu \mathrm{mol} / \mathrm{L})$, and platelet activability was quantified with a FacScan flow cytometer (Becton Dickinson, Mountain View, Calif). Saturating concentrations of different monoclonal antibodies directly coupled with fluorochromes were used: SZ2 directed against GP 1b $\alpha$, P2 directed against GP $\alpha \mathrm{IIb} \beta 3$ complex (Beckman Coulter), and monoclonal antibody anti-P-selectin (anti-CD62P, Becton Dickinson). The results are expressed as a percentage of positive platelets for CD62P expression or as the number of GP sites using calibration beads (Byocytex, Marseille, France). Normal values from the laboratory were previously recorded from 600 control subjects for factor I levels, von Willebrand factor (antigen and activity), and platelet integrins. 


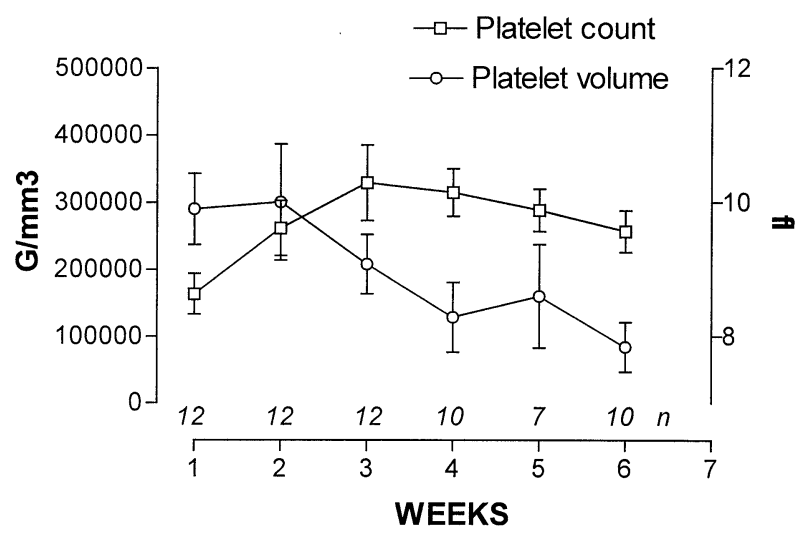

Figure 1. Time course of platelet volume (fl) and platelet count after external VAD implantation. ( ${ }^{*} P<.05$ vs laboratory control values; $\mathbf{n}=$ absolute number of patients.)

\section{Statistical Analysis}

The results are expressed as mean $\pm \mathrm{SD}$ or median (extremes). Flow cytometric measurement of integrin expression was compared using the Mann-Whitney $U$ test, and correlation with aspirin doses was calculated with ranked patient data using the Spearman test.

\section{Results}

\section{Patients}

Two patients were female. The median age was 44.3 years (16.6-58.6 years). Mechanical support was biventricular in 10 patients (5 had a left ventricular support only). The median CPB duration was 162 minutes (96-339 minutes), and the median duration of support was 72 days (28-360 days). Three patients died during VAD support. The first patient died of sepsis, the second patient died of intracranial hemorrhage, and the third patient died of device dysfunction.

\section{Platelet Count and Platelet Volume}

Figure 1 shows the platelet count and volume on weekly follow-up. The platelet count steadily increased during the first 2 postoperative weeks and remained stable after the fourth postoperative week (laboratory control values are $150-400 \mathrm{giga} / \mathrm{mm}^{3}$ and 8-12 fl for platelet count and platelet volume, respectively). A type II heparin-induced thrombocytopenia developed in 1 patient the fourth week after device implantation. Heparin was stopped and replaced by antivitamin $\mathrm{K}$ therapy. Aspirin was maintained.

\section{Resistance to Aspirin Treatment}

Figure 2 represents platelet aggregation velocity in patients treated with aspirin. The zero y axis value corresponds to the absence of platelet aggregation (and aspirin efficacy).

The mean doses of aspirin administered per day (day of blood collection for platelet ex vivo testing) were the fol-

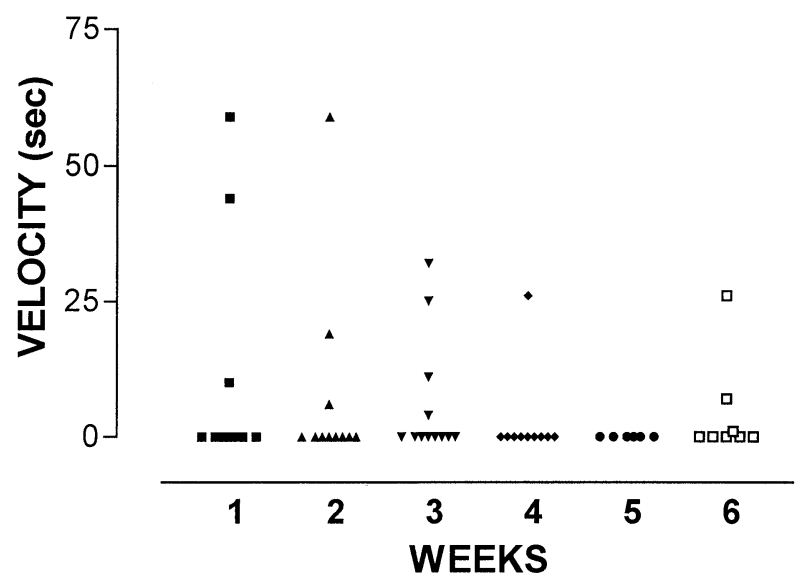

Figure 2. Ex vivo platelet aggregation velocity in the presence of AA $(0.2 \mathrm{mg} / \mathrm{mL})$ in patients receiving aspirin. Resistance to aspirin treatment was defined as any persisting ex vivo platelet aggregation induced by AA despite a daily administration of aspirin.

lowing: first week, $260 \pm 38 \mathrm{mg}$; second week, $249 \pm 90$ $\mathrm{mg}$; third week, $255 \pm 100 \mathrm{mg}$; fourth week, $250 \pm 149$ $\mathrm{mg}$; fifth week, $273 \pm 160 \mathrm{mg}$; and sixth week, $288 \pm 164$ mg.

Aspirin resistance, as previously defined, was observed in a total of 6 patients. These patients are represented in Figure 2 by any value above the zero value of platelet aggregation velocity at each weekly blood test.

Five of 6 patients responded to an increased daily dose of aspirin. Three of 5 patients had a recurrence of this phenomenon when the daily dose of aspirin was increased.

In 3 patients, aspirin resistance was observed from the first week of treatment with aspirin after VAD implantation.

\section{Inflammatory State: Endothelial Activation}

Figure 3 shows the temporal trend of fibrinogen levels significantly above laboratory control values (2-4 g/L) during the entire postoperative course.

Figure 4 displays the temporal trend of circulating von Willebrand factor (activity and antigenic determination) during the postoperative course. These levels remained significantly above laboratory control values $(60 \%-100 \%$ von Willebrand factor activity and 60\%-100\% antibody binding) after implantation and during the entire study period.

\section{Platelet Activation}

The expression of P-selectin on the membrane surface of platelets was present during the entire study period (Figure 5). The normal range value was 3000 to 6500 sites per platelet.

Figure 6 shows the expression of GP $\alpha \mathrm{IIb} \beta 3$ and GP Ib $\alpha$ during the postoperative course. The normal range values were 24,000 to 38,000 and 10,500 to 19,000 sites per platelet for GP $\alpha \operatorname{IIb} \beta 3$ and GP Ib $\alpha$, respectively. After the 


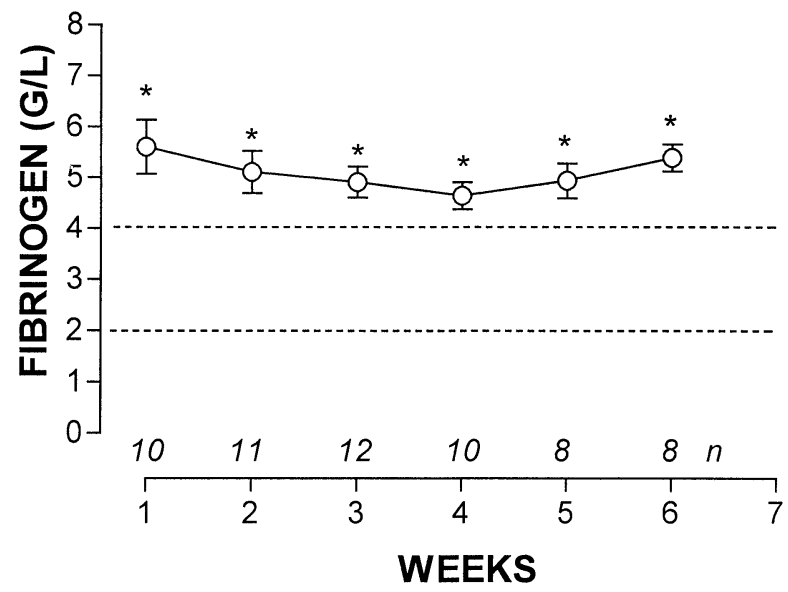

Figure 3. Time course of fibrinogen levels after external VAD implantation. Laboratory control values (dotted lines). $\left({ }^{*} P<.05\right.$ vs laboratory control values; $\mathbf{n}=$ absolute number of patients.)

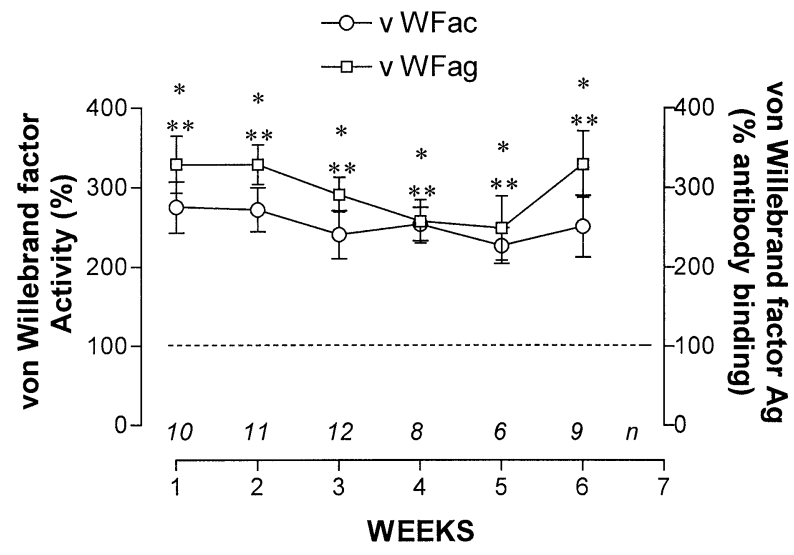

Figure 4. Time course of circulating von Willebrand factor antigen and activity after external VAD implantation. Laboratory control values (dotted lines). $\left({ }^{*} \boldsymbol{P}<.05\right.$ and ${ }^{* *} \boldsymbol{P}<.05$ : von Willebrand factor [antigen and activity] vs laboratory control values; $\mathbf{n}=$ absolute number of patients.) v WFac; Von Willebrand factor activity; $v$ WFag, von Willebrand factor antigen.

first week, GP $\alpha \operatorname{IIb} \beta 3$ expression remained within the normal range but closer to the lower limit. GP Ib $\alpha$ expression remained in the lower limits but closer to normal range values. Ex vivo-induced externalization of GP $\alpha \operatorname{IIb} \beta 3$ and internalization of GP Ib $\alpha$ in the presence of adenosine remained within normal values during the entire postoperative course (Figures 7 and 8) (normal range values are 26\% $\pm 11 \%$ for GP $\alpha \operatorname{IIb} \beta 3$ externalization and $17 \% \pm 9 \%$ for GP Ib $\alpha$ internalization).

No difference was observed in the expression of GP $\alpha \mathrm{IIb} \beta 3$ and GP Ib $\alpha$ when comparing patients who displayed some resistance to aspirin and other patients. No correlation was observed between the daily aspirin dose and the expression of integrin on platelet surface by flow cytometry.

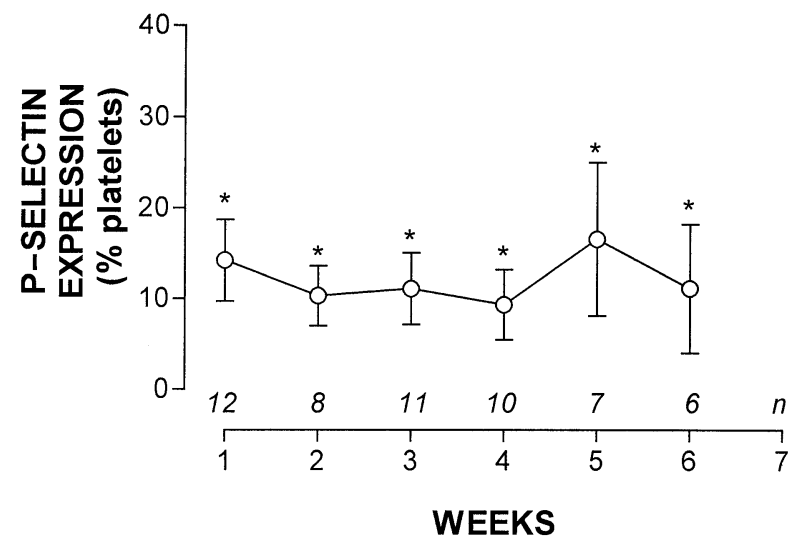

Figure 5. Time course expression of P-selectin on platelet surface membrane after external VAD implantation from flow cytometry measurements. Laboratory control value is less than $2 \%$ of platelets expressing this surface protein. ( ${ }^{*} P<.05$ vs laboratory control values; $\mathbf{n}=$ absolute number of patients.)

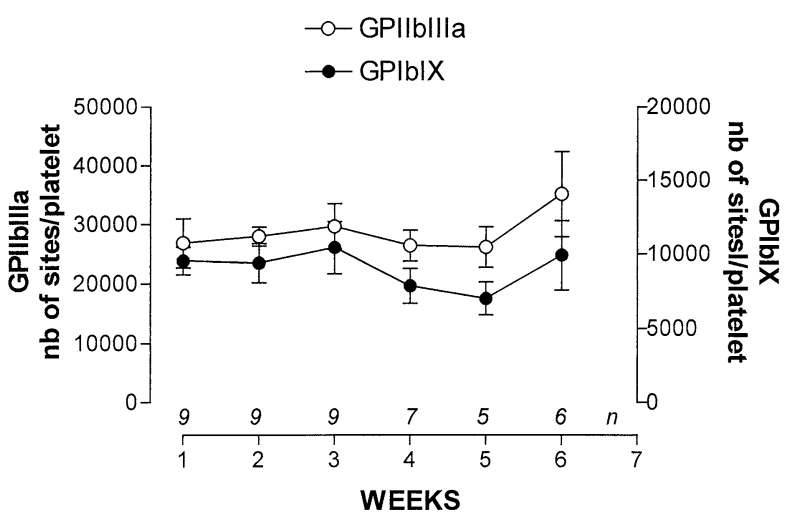

Figure 6. Time course expression of GPs $\alpha \mathrm{llb} \beta 3$ and Ib $\alpha$ on platelet surface membrane after external VAD implantation from flow cytometry measurements. No difference was observed when compared with laboratory control values $(n=$ absolute number of patients). GP, Glycoprotein.

\section{Discussion}

This study examined platelet activation and aggregation in patients implanted with an external pulsatile VAD for primary cardiogenic shock. We observed a persisting platelet activation profile and normal or reduced platelet activability after implantation. We also observed a resistance to aspirin in $26 \%$ of the patients who responded to increased doses of aspirin up to $500 \mathrm{mg} / \mathrm{d}$.

In healthy subjects, aspirin efficacy is observed with low doses $(<100 \mathrm{mg} / \mathrm{d})$, but with some individual variability. ${ }^{1,2}$ In patients with atherosclerotic disease, its efficacy is reduced and may necessitate higher daily doses to overcome an erythrocyte promotion of platelet reactivity. ${ }^{4,12,13}$ After $\mathrm{CPB}$, thromboxane $\mathrm{A}_{2}$ synthesis persists at $70 \%$ to $54 \%$ of 
control values after 5 and 10 days, respectively, despite a daily oral intake of $100 \mathrm{mg}$ of aspirin. ${ }^{3}$

We observed a persistent AA-induced platelet aggregation in the presence of AA in $26 \%$ of patients receiving at least $250 \mathrm{mg}$ of aspirin daily. Moreover, this so-called aspirin resistance could occur a few weeks after surgery, whereas previous platelet studies showed an ex vivo aspirin efficacy in the same patient. The mechanism of this phenomenon may be explained by an increased turnover of platelets in the early postoperative weeks, as observed after $\mathrm{CPB}$. This increased turnover of platelets may overcome the effect of aspirin on thromboxane $\mathrm{A}_{2}$ synthesis. ${ }^{3} \mathrm{An}$ increased turnover may be a possibility in our patients with implanted VADs. In our study, we observed an increased platelet volume with a decreased platelet count during the early postoperative weeks after VAD implantation with CPB.

Another mechanism of resistance to aspirin could be an inflammatory-induced cyclooxygenase- 2 isoform activity that is less sensitive to aspirin acetylation. ${ }^{5}$ Indeed, a prolonged inflammatory reaction is observed in patients implanted with VADs. Cytokine levels such as interleukin-6 and interleukin-8 or complement fractions (C3a, C5a) are increased several weeks after implantation. ${ }^{14,15}$ These molecules have also been reported to induce platelet activation. ${ }^{16}$ A persistent inflammatory state was also observed in our patients, as expressed by elevated fibrinogen levels and circulating von Willebrand factor values during the postoperative course.

Platelet activation studies measuring the membrane expression of P-selectin showed a persistent activation of platelets during this prolonged period of support. ${ }^{17}$ In vitro, the GP $\alpha \operatorname{IIb} \beta 3$ complex has been reported to be selectively degraded after prolonged ventricular assist. ${ }^{18}$ In our patients, integrin $\alpha \operatorname{IIb} \beta 3$ and $\mathrm{Ib} \alpha$ expression on platelet surface remained within normal values and may reflect a persisting moderate platelet activation. No correlation was found between the daily dose of aspirin and membrane expression of these GPs, and no difference was observed between the integrin expression in patients who displayed resistance to aspirin and other patients. Membrane expression of integrin $\alpha \mathrm{IIb} \beta 3$ has individual variations according to gender or genetic polymorphism associated with different aspirin sensitivity. ${ }^{19-21}$ This persistent platelet activated state could be related to permanent activation from a pathway other than the ADP pathway: Continuous thrombin generation has been reported in patients implanted with different VADs. ${ }^{15,22,23}$ Thrombin has a powerful effect on platelet activation through specific protease-activated receptor pathways (1 and 4) insensitive to aspirin. ${ }^{24-26}$

GP Ib $\alpha$ is coupled with von Willebrand factor when activated $^{9,27}$ by high shear stress stimuli and is responsible for platelet adhesion and activation. This pathway is poorly

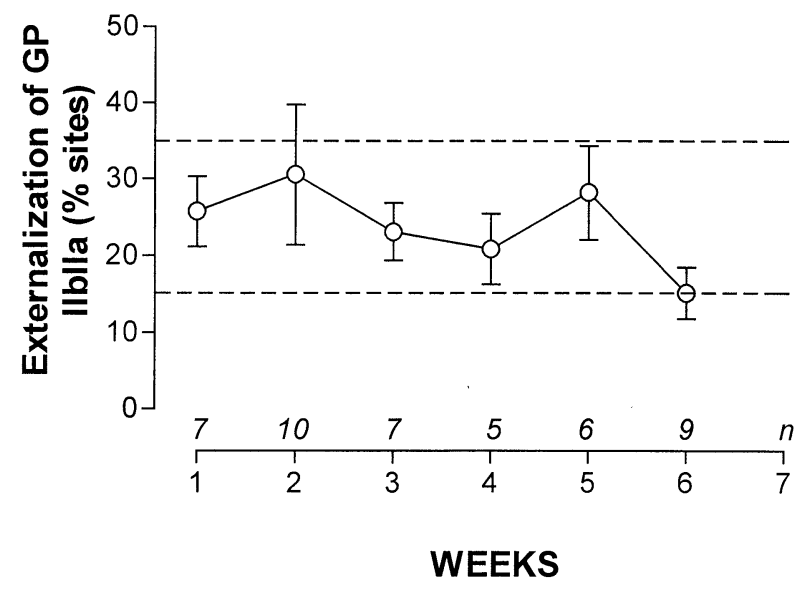

Figure 7. Time course externalization of GP $\alpha \mathrm{llb} \beta 3$ on platelet surface membrane after ex vivo induction using ADP (25 $\mu \mathrm{mol} / \mathrm{L})$ ( $\mathbf{n}=$ absolute number of patients). Laboratory control values (dotted lines). GP, Glycoprotein.

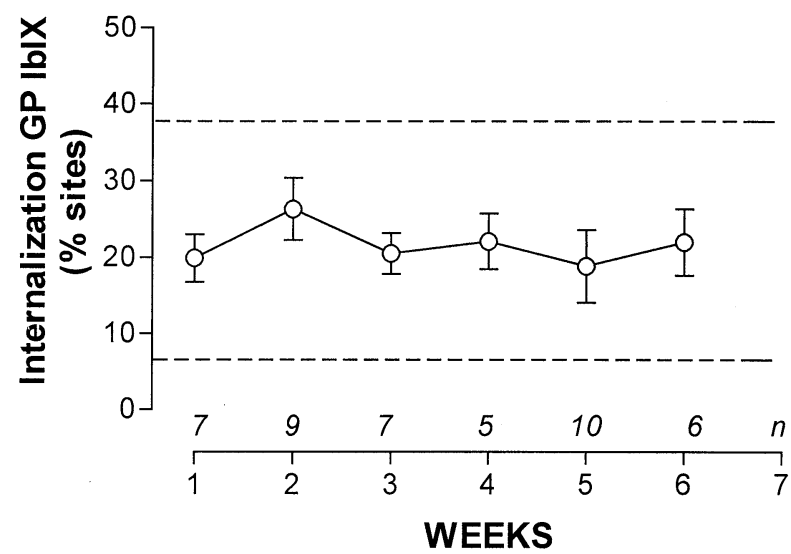

Figure 8. Time course externalization of GP Ib $\alpha$ on platelet surface membrane after ex vivo induction using ADP (25 $\mu \mathrm{mol} / \mathrm{L})$ ( $\mathrm{n}$ $=$ absolute number of patients). Laboratory control values (dotted lines). GP, Glycoprotein.

sensitive to aspirin. ${ }^{26,28}$ The platelet activation observed in our patients could therefore be explained by high shear stress in the presence of an artificial ventricle. High shear stress could be developed by a high upstroke pattern on the apex cardiogram in the artificial ventricle with reduced inflow and outflow conduit diameters (14 $\mathrm{mm}$ diameter), resulting in high conduit velocities. Moreover, the substrates of these receptors (von Willebrand factor) remained within high-range values during the 6 postoperative weeks, indicating facilitated receptor-substrate interactions. These mechanisms of platelet activation make the efficacy of aspirin as an antiplatelet agent questionable despite the observation of decreased thromboxane $\mathrm{A}_{2}$ production.

These patients are also characterized by a preserved platelet reactivity considering only GP $\alpha \operatorname{IIb} \beta 3$ and GP Ib $\alpha$ 
(Figures 6 and 7), despite an initial increased turnover associated with a continuous activation. The balance between enhanced platelet activation and reactivity may contribute to the individual tendencies for thrombosis or bleeding in patients with this type of VAD. Aspirin may favor the balance toward a reduced platelet aggregation but requires appropriate monitoring and adjusted doses to be biologically efficient.

\section{References}

1. Pappas JM, Westengard JC, Bulls JS. Population variability in the effect of aspirin on platelet function. Implications for clinical trials and therapy. Arch Pathol Lab Med. 1993;118:801-4.

2. Gum PA, Kottke-Marchant K, Poggio ED, Gurm H, Welsh PA, Brooks L, et al. Profile and prevalence of aspirin resistance in patients with cardiovascular disease. Am J Cardiol. 2001;88:230-5.

3. Zimmerman N, Kienzle P, Weber AA, Winter J, Gams E, Schrör K, et al. Aspirin resistance after coronary artery bypass grafting. $J$ Thorac Cardiovasc Surg. 2001;121:982-4.

4. Valles J, Santos T, Aznar J, Osa A, Lago A, Cosin J, et al. Erythrocyte promotion of platelet reactivity decrease the effectiveness of aspirin as an antithrombotic therapeutic modality. Circulation. 1998;97:350-5.

5. Weber A-A, Zimmerman KC, Meyer-Kichrath J, Schrör K. Cyclooxygenase-2 in human platelets as a possible factor in aspirin resistance. Lancet. 1999;353:900.

6. Baker LC, Davis WC, Autieri J, Watach MJ, Yamazaki K, Litwak P, et al. Flow cytometric assays to detect platelet activation and aggregation in device-implanted calves. J Biomed Mater Res. 1998;41:31221.

7. Abhrams CS, Ellison N, Budzynski AZ, Shattil SJ. Direct detection of activated platelets and platelet-derived microparticles in humans. Blood. 1990;88:1525-41.

8. Kamath S, Blann AD, Lip AHY. Platelet activation: assessment and quantification. Eur Heart J. 2001;22:1561-71.

9. Goto S, Ikeda Y, Saldivar E, Ruggeri ZM. Distinct mechanisms of platelet aggregation as a consequence of different flows conditions. J Clin Invest. 1998;101:479-86.

10. Houel R, Moczar M, Clerin V, Loisance DY. Pseudointima in inflow conduits of left ventricular assist devices. Ann Thorac Surg. 1999;68: 717-23.

11. El-Banayosy A, Arusoglu L, Kzner L, Tenderich G, Minami K, Inoue $\mathrm{K}$, et al. Novacor left ventricular assist system versus HeartMate vented electric left ventricular assist system as a long term mechanical circulatory support device in bridging patients: a prospective study. J Thorac Cardiovasc Surg. 2000;119:581-7.

12. Santos T, Valles J, Aznar J, Marcus AJ, Broekman MJ, Safier LB.
Prothrombotic effects of erythrocytes on platelet reactivity. Circulation. 1997;95:63-8.

13. Helgason CM, Bolin KM, Hoff JA, Winkler SR, Mangat A, Tortori $\mathrm{KL}$, et al. Development of aspirin resistance in person with previous ischemic stroke. Stroke. 1994;25:2331-6.

14. Corry DC, DeLucia A, Zhu H, Radcliffe RR, Brevetti GR, El-Khatib $\mathrm{H}$, et al. Time course of cytokine release and complement activation after implantation of the HeartMate left ventricular assist device. ASAIO J. 1998;44:M347-51.

15. Wilhem CR, Ristish J, Kormos RL, Wagner WR. Monocyte tissue factor expression and ongoing complement generation in ventricular assist device patients. Ann Thorac Surg. 1998;65:1071-6.

16. Lumadue JA, Lanzkron SM, Kennedy SD, Khul DT, Kikkler TS. Cytokine induction of platelet activation. Am J Clin Pathol. 1996;106: 795-8.

17. Matsubayashi H, Fastenau DR, McIntyre JA. Changes in platelet activation associated with left ventricular assist system placement. J Heart Lung Transplant. 2000;19:462-8.

18. Serna DL, King BO, Huh J, Jalal R, Tran L, Chen JC. Evidence for selective degradation of platelet GP IIb/IIIa complex after prolonged in vitro ventricular assist. J Invest Med. 1998;46:279-83.

19. Weiss EJ, Bray PF, Tayback M. A polymorphism of platelet glycoprotein receptor as an inherited risk factor for coronary thrombosis. N Engl J Med. 1996;334:1090-4.

20. Faraday N, Goldschmidt-Clermont P, Bray PF. Gender differences in platelet GPIIb-IIIa activation. Thromb Haemost. 1997;77:748-54.

21. Cooke GE, Bray PF, Hamlington JD, Pham DM, Goldsmidt-Clermont PJ. PIA2 polymorphism and efficacy of aspirin. Lancet. 2001;351: 1253.

22. Spanier T, Oz M, Levin H, Weinberg A, Stamatis K, Stern D, et al. Activation of coagulation and fibrinolytic pathways in patients with left ventricular assist devices. J Thorac Cardiovasc Surg. 1996;112: 1090-7.

23. Koster A, Loebe M, Hanse R, Potapov EV, Noon GP, Kuppe H, et al. Alterations in coagulation after implantation of a pulsatile Novacor LVAD and the axial flow Micromed DeBackey LVAD. Ann Thorac Surg. 2000;70:533-7.

24. Kahn ML, Zheng Y, Huang W, Bigornia V, Zeng D, Moff S, et al. A dual thrombin receptor system for platelet activation. Nature. 1998; 394:690-4.

25. Brass S. Platelets and proteases. Nature. 2001;413:26-7.

26. Parker RI, Gralnick HR. Effect of aspirin on platelet-von Willebrand factor surface expression on thrombin and ADP-stimulated platelets. Blood. 1989;74:2016-21.

27. Kroll MH, Hellums JD, McIntire LV, Shafer AI, Moake JL. Platelets and shear stress. Blood. 1996;88:1525-41.

28. Maalej N, Folts JD. Increased shear stress overcome the antithrombotic platelet inhibitory effect of aspirin in stenosed dog coronary artery. Circulation. 1996;93:1201-5. 